\title{
Misfire Fault Diagnosis Method for Diesel Engine Based on MEMD and Dispersion Entropy
}

\author{
Cheng Gu $\mathbb{D}$, Xin-Yong Qiao $\mathbb{D}$, Huaying Li $\mathbb{D}$, and Ying Jin $\mathbb{C}$ \\ Department of Vehicle Engineering, Academy of Army Armored Forces, Beijing, China \\ Correspondence should be addressed to Xin-Yong Qiao; qxyaafe@sina.com
}

Received 25 November 2019; Accepted 18 January 2021; Published 2 February 2021

Academic Editor: Cristina Castejón

Copyright (c) 2021 Cheng Gu et al. This is an open access article distributed under the Creative Commons Attribution License, which permits unrestricted use, distribution, and reproduction in any medium, provided the original work is properly cited.

\begin{abstract}
As a main source of power, diesel engines are widely used in large mechanical systems. Fire failure is a kind of common fault condition, which seriously affects the power and economy of the diesel engine. Previously, scholars mostly used single-channel signal to diagnose the misfire fault of the diesel engine. However, the single-channel signal has limitations in reflecting the information of fault. A novel fault diagnosis method based on MEMD and dispersion entropy is proposed in this paper. Firstly, the multichannel vibration signal of the diesel engine cylinder head is decomposed by multivariate empirical mode decomposition (MEMD), which obtains the IMF component groups with the same frequency in the same order. Then, the IMF component with a large correlation coefficient with the original signal in each group is selected to reconstruct new signal, and dispersion entropy (DE) of the reconstructed signal is calculated as a fault feature vector. Finally, the fault feature vector is input into the support vector machine (SVM) for misfire fault classification. Compared with the other three methods, the results show that the diagnosis method proposed in this paper can effectively extract the fault features and accurately identify the fault type, which is superior to the comparison method.
\end{abstract}

\section{Introduction}

Diesel engine is the main power mechanism for a heavy equipment, whose working conditions determine the reliability and safety of the whole system. Misfire is a common failure state of diesel engine, which attracts an increasing amount of attention because it can cause serious deterioration on the power and economic performance of engine [1]. It is of great significance to diagnose misfire fault quickly and accurately for the normal and stable operation of the equipment.

At present, there are many methods for diagnosing diesel engine failures, such as vibration detection method, cylinder pressure detection method, instantaneous rotation speed detection method, and oil detection method [2-5]. The vibration signal of cylinder head is widely concerned because of its rich information and easy access. However, due to the complicated structure of diesel engine and many moving parts, the vibration signal of cylinder head mixes the signals of different frequency excitation sources and strong noise, which makes it nonstationary and nonlinear [6]. It is the key to extract the fault feature from the complex vibration signal for the state recognition.

Traditional signal analysis method mainly contains time domain analysis and frequency domain analysis [7], but only for stationary signal. Considering the nonlinear nonstationary characteristics of diesel engine vibration signal, traditional signal methods cannot effectively extract fault feature information. In recent years, some new signal processing methods have been introduced, such as wavelet transform (WT) [8], wavelet packet transform (WPT) [9], and empirical mode decomposition (EMD) [10]. The EMD decomposes the signal into a series of IMF components that reflect the signal frequency based on the characteristics of the signal itself. Compared with wavelet transform, empirical mode decomposition overcomes the defects of its fixed basis function. Due to its powerful ability to adaptively decompose signals, it is widely used in mechanical, biomedical, weather, and financial data analysis and other fields [11-14]. 
The EMD method is only suitable for processing onedimensional signals. The multivariate signal can be divided into multiple one-dimensional signals after processed by EMD, which may cause the problem of different amount of IMF components obtained after decomposition, or inconsistent frequency corresponding to the same order IMF components. . It does not make any sense for signal analysis. However, signals acquired by multichannel are most commonly used in engineering practice, so it is crucial to come up with a new approach for multivariate signal analysis. Rilling et al. proposed bivariate EMD in 2007 [15], which uses the complex signal as a superposition of two kinds of rotational components, fast and slow, and decomposes by extracting the rotational component in three-dimensional space, considering mutual information and morphological logic between real and imaginary parts. The physical meaning of each component after decomposing is clearly defined. For the three-dimensional signal, the trivariate EMD method was proposed by Ur Rehman and Mandic [16], which projects the multivariate signal on the threedimensional spherical direction vector and calculates the mean value. In the later research, Rehman proposed multivariate empirical mode decomposition (MEMD) for highdimensional signal [17]. It projects multidimensional signals to hyperspherical direction vector of multidimensional space, calculates the mean value of envelope of each signal, and obtains the local mean value of multivariate signals to realize the same mode decomposition of the multisignal in different frequency bands, which can decompose signals of any dimension. The MEMD algorithm realizes multichannel signal simultaneous joint analysis to ensure that the modes of different channels are the same, that is, the IMF components match and align in quantity and scale, which solves the problem of multichannel signal mode calibration.

Nonlinear analysis has been widely used in fault diagnosis of various mechanical equipment as a feature extraction method. The nonlinear analysis method includes Lyapunov exponent [18], fractal dimension [2], and information entropy [19]. Information entropy is used to represent the uncertainty of the system and a variety of entropy values are developed to describe the nonlinear characteristics of the system. In 1991, Pincus [20] proposed the concept of approximate entropy (ApEn), which characterizes the complexity of dynamical systems with time series dimensional changes. Subsequently, Richman and Moorman [21] improved the approximate entropy and proposed the sample entropy algorithm (SE), but the sample entropy has the defects of slow calculation and poor real-time performance, and the similarity measurement is prone to mutation; Bandt and Pompe [22] introduced permutation entropy (PE). Although the concept of permutation entropy is simple and the calculation speed is fast, the difference of amplitude of signal is not considered. Rostaghi and Azami [23] proposed a new method to measure the complexity of time series, namely, dispersion entropy (DE), which solved the deficiency of sample entropy and permutation entropy.

In this paper, MEMD is used to decompose the multichannel signal, and reconstructed signal is composed of the
IMF component with a large correlation coefficient with the original signal. The DE value of the reconstructed signal is used as the fault feature vector to evaluate the diesel engine state and fault diagnosis. This paper is organized as follows. Research background and significance are introduced in Section 1. In Section 2, the MEMD theory is introduced and comparison between EMD and MEMD is carried out with simulation signal. In Section 3, DE algorithm is introduced and the effect of parameter on DE is analyzed. In Section 4 and Section 5, the scheme of fault diagnosis is introduced and the different fault is recognized by vibration signals of four states of diesel engine. The conclusion is given in Section 6 .

\section{Multivariate Empirical Mode Decomposition}

2.1. EMD. Empirical mode decomposition is a data-driven method that can process nonlinear and nonstationary signals adaptively, which eliminates the limitation based on Fourier transform. The main idea of EMD is to decompose a univariate signal into several oscillatory modes called intrinsic mode function (IMF). Two conditions need to be satisfied when the IMF generates: (1) in the whole dataset, the number of extrema and the number of zero crossings must be equal or differ at most by one, (2) at any point, the mean of the upper and lower envelopes must be zero. The detailed procedure is showed in Algorithm 1.

2.2. $M E M D$. In engineering application, univariate signal behaves inferior due to the small amount of information contained, it cannot reflect the characteristics of the system completely. With the development of sensor technology, the method of obtaining signals by multiple sensors is gradually developed. However, there is uncertainty problem of scale arrangement in the decomposition of EMD, which affects the results of characteristic analysis. In order to solve this problem, the multivariate empirical mode decomposition was proposed by Rehman and Mandic [17] in 2009, which can realize the same mode analysis of multiple signals in different frequency scales. Considering the fact that the local mean of the $n$-dimensional signal cannot be directly defined, MEMD obtains a sequence of projection vectors along different directions in the $n$-dimensional, and then the local mean of each direction is obtained. The number of IMFs of each channel signal is equal after decomposition, which realizes the alignment of frequency scales of each IMF corresponding to each channel. The detailed procedure is showed in Algorithm 2.

2.3. Comparison between EMD and MEMD. In order to verify the decomposition effect of MEMD on multichannel signals, simulated multivariate signal composed of different frequency is built up. The frequencies are as follows: $f_{1}=10 \mathrm{~Hz}, f_{2}=50 \mathrm{~Hz}$, and $f_{3}=200 \mathrm{~Hz}$, and Gaussian noise is added to each channel to reflect working condition accurately, as shown in equation (1). The sampling frequency is 
(1) Find all local extrema of the original signal $x(t)$; fit the upper envelope $u(t)$ and the lower envelope $v(t)$ according to the cubic spline function, $v(t) \leq x(t) \leq u(t)$.

(2) Calculate the local mean from the upper and lower envelopes $m(t)=[u(t)+v(t)] / 2$, and then subtract it from $x(t), h_{1}(t)=m(t)-$ $x(t)$.

(3) Check whether $h_{1}(t)$ satisfies the two conditions. If satisfies, $h_{1}(t)$ is the first IMF component; otherwise, replace $h_{1}(t)$ with $x(t)$ and repeat (1)-(2) until the termination criterion is satisfied. Then, set the first IMF as $c_{1}(t)$.

(4) Repeat (1)-(3) until all the IMFs and the residual are extracted. Finally, the original signal $x(t)$ can be decomposed as $x(t)=\sum_{i=1}^{n} c_{i}(t)+r_{n}(t)$.

Algorithm 1: The standard EMD algorithm.

(1) Choose a uniform sampling point set on (n-1)-dimensional by the Hammersly sequence, and then establish an $n$-dimensional spatial direction vector.

(2) Calculate the projection of the input signal $\{\mathbf{v}(t)\}_{t=1}^{T}$ along all the directions $x^{\theta_{k}}$, and then obtain the projection set $\left\{p^{\theta_{k}}(t)\right\}_{k=1}^{K}$.

(3) Find the maxima in each projection direction $\left\{\mathbf{v}\left(t_{j}^{\theta_{k}}\right)\right\}$ and its corresponding moment, and then calculate the multivariate envelope curves $\left\{e^{\theta_{k}}(t)\right\}_{k=1}^{K}$ by spline interpolation function.

(4) For a set of $K$ direction vectors, calculate the mean of the envelope curves by $\mathbf{m}(t)=\sum_{k=1}^{K} \mathbf{e}^{\theta_{k}}(t) / K$.

(5) The remaining value is subtracted as $\mathbf{c}_{i}(t)=\mathbf{v}(t)-\mathbf{m}(t)$, and check whether $\mathbf{c}_{i}(t)$ satisfies the stoppage criterion. If satisfies, $\mathbf{c}_{i}(t)$ is the first IMF component and apply (2) (5) to $\mathbf{v}(t)-\mathbf{c}_{i}(t)$; otherwise, apply it to $\mathbf{c}_{i}(t)$. Finally, the multivariate signal can be decomposed as $\mathbf{v}(t)=\sum_{i=1}^{d} \mathbf{c}_{i}(t)+\mathbf{r}(t)$.

Algorithm 2: The multivariate EMD algorithm.

set to $2048 \mathrm{~Hz}$, and the sampling time is set to $1 \mathrm{~s}$. The wave

of simulation signal is shown as Figure 1.

$$
\left\{\begin{array}{l}
x_{1}=0.6 \sin \left(2 \pi f_{1} t\right)+\sin \left(2 \pi f_{2} t\right)+0.8 \cos \left(2 \pi f_{3} t\right)+0.1 \mathrm{rand} n(1, N), \\
x_{2}=\sin \left(2 \pi f_{1} t\right)+\sin \left(2 \pi f_{2} t\right)+0.5 \cos \left(2 \pi f_{3} t\right)+0.1 \operatorname{rand} n(1, N), \\
x_{3}=0.4 \sin \left(2 \pi f_{1} t\right)+1.2 \sin \left(2 \pi f_{2} t\right)+0.8 \cos \left(2 \pi f_{3} t\right)+0.1 \operatorname{rand} n(1, N) .
\end{array}\right.
$$

The simulated multivariate signal is decomposed by EMD and MEMD, as shown in Figures 2 and 3, respectively. The multisignal processing method by EMD uses the signal of each channel as an independent single signal for empirical mode decomposition. After the decomposition by two methods, a set of IMF components are extracted from original signal; the following observations can be made:

(1) Whether using EMD or MEMD, the IMF components are divided into three groups, and each group of IMF components corresponds to the signal of respective channel.

(2) The number is different between IMF components obtained by EMD and IMF components obtained by MEMD. Furthermore, the number of IMF components obtained by EMD is distinct in each channel. It is easy to see from Figure 2 that $x_{1}$ and $x_{3}$ are decomposed by EMD into 8-IMF components, while $x_{2}$ only decomposes into 7 . The reason is that $x_{1}, x_{2}$, and $x_{3}$ are treated as independent individuals, without considering the internal correlation, while MEMD overcomes it.

(3) The same characteristic frequencies appear in the same order after MEMD, while the phenomenon does not occur after EMD, which illustrates that MEMD can extract the same oscillation mode from multisignals and be conducive to signal analysis further.

\section{Dispersion Entropy}

The reconstructed signal composed of IMF components with large correlation coefficient filtered by MEMD reflects the fault characteristics effectively. It is crucial to extract the fault feature from the reconstructed signal. When a cylinder of diesel engine fails, the complexity of vibration signal on cylinder cover changes due to the evenness of the cylinder burst. Therefore, this paper proposes to extract feature by dispersion entropy from multichannel signal. 


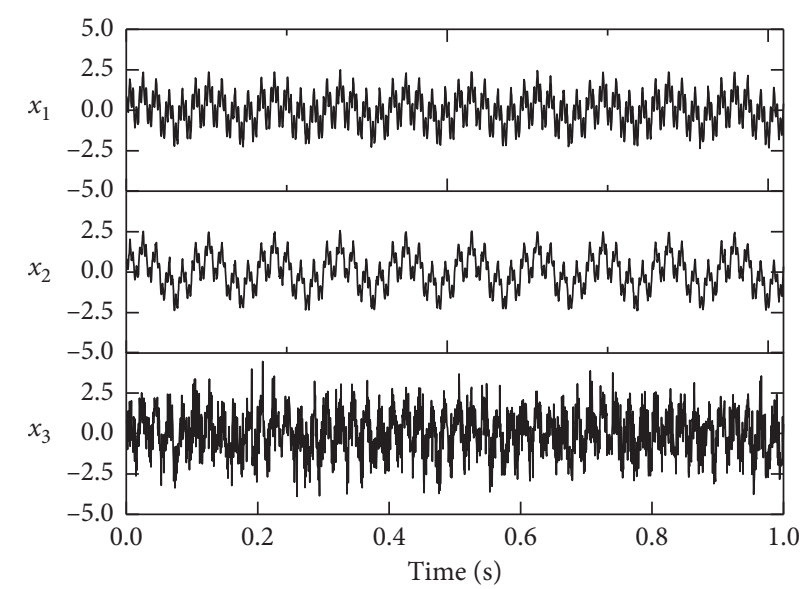

FIGURE 1: The original signal of simulation.

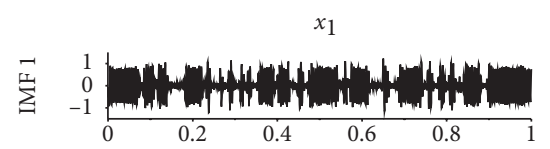

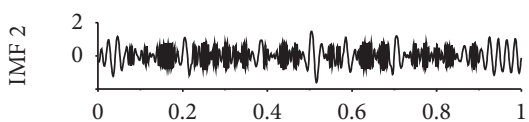

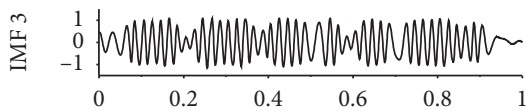

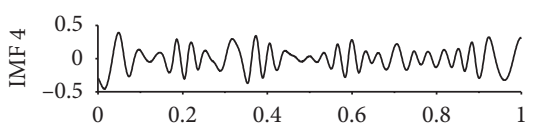

$\sum_{0}^{\infty} 0 \underbrace{0.4}_{0.2} 0_{0.6}^{0.8} 1$
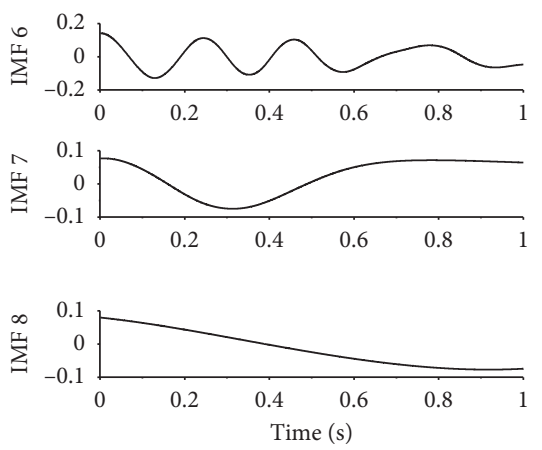

(a)

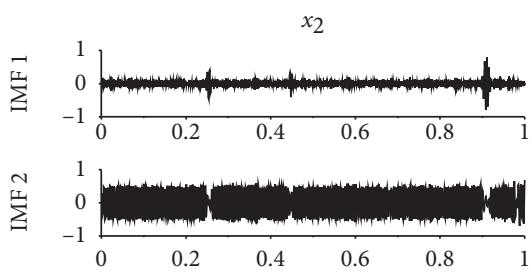

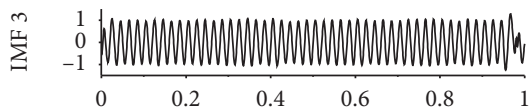
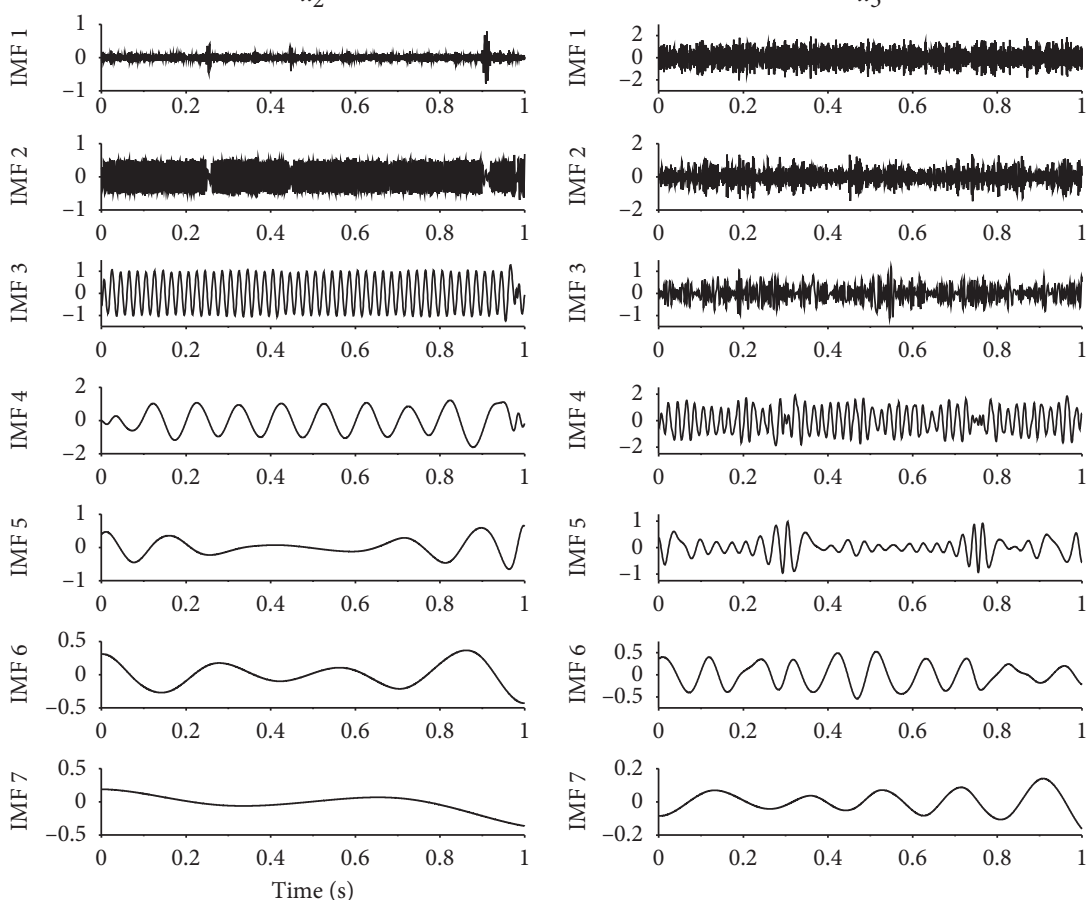

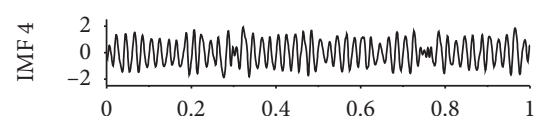

$\sum_{-1}^{n} \underbrace{1}_{0.2} \begin{array}{llll}0.4 & 0.6 & 0.8\end{array}$
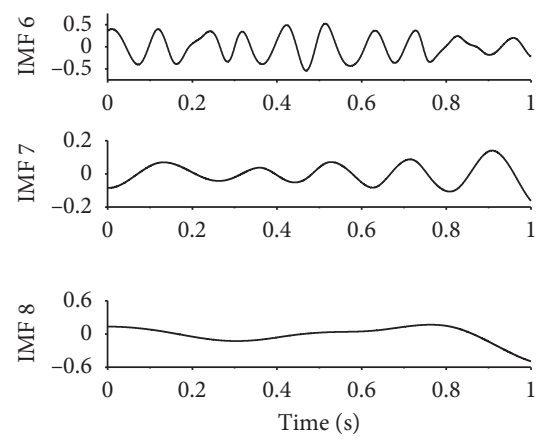

(c)

Figure 2: EMD-based decomposition of simulation signal.

Dispersion entropy (DE) is a new algorithm for measuring time series complexity proposed by Rostaghi and Azami in 2016 [23]. It overcomes the defect that the permutation entropy does not consider the magnitude of the amplitude with good stability and fast calculation speed. For a time series $x=\left\{x_{1}, x_{2}, \cdots, x_{N}\right\}$ with the length of $N$, the calculation process of $\mathrm{DE}$ is as follows:
(1) The normal cumulative distribution function (NCDF) is employed to map $\mathbf{x}$ to $\mathbf{y}$ from 0 to 1 :

$$
y_{j}=\frac{1}{\sqrt{2 \pi} \sigma} \int_{-\infty}^{x_{j}} e^{-\left((t-\mu)^{2} / 2 \sigma^{2}\right) d t}
$$

(2) A linear algorithm is used to map $\mathbf{y}$ to the range of $[1,2, \cdots, c]$ and get a sequence $z_{j}^{(c)}$ : 

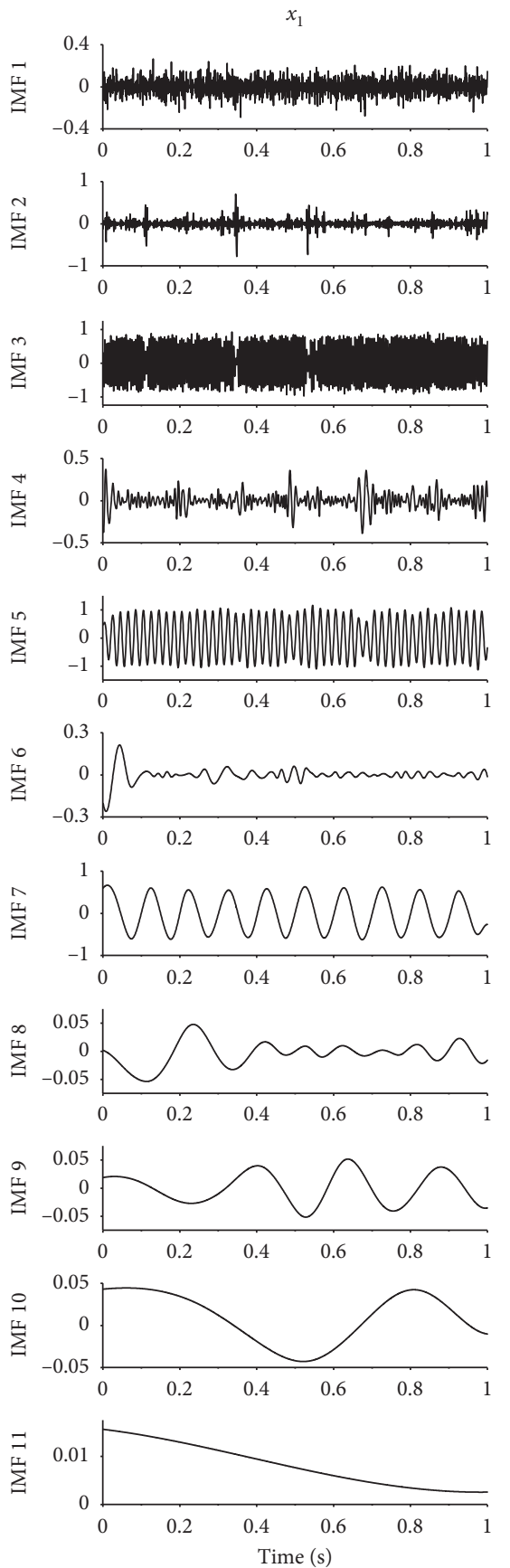

(a)
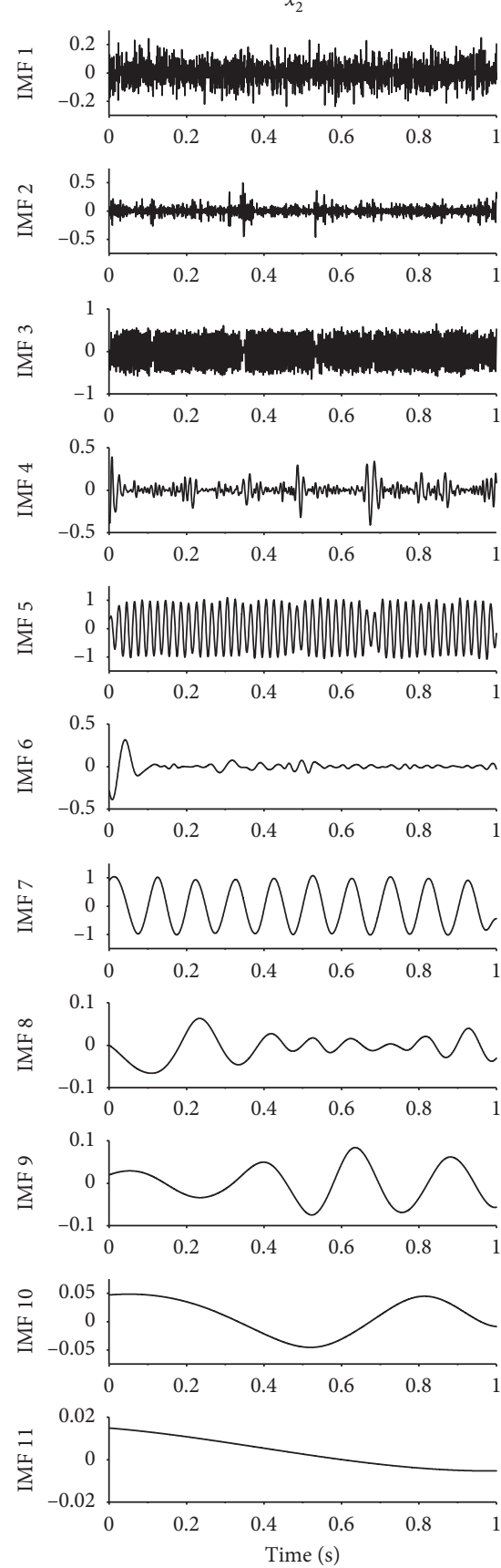

(b)
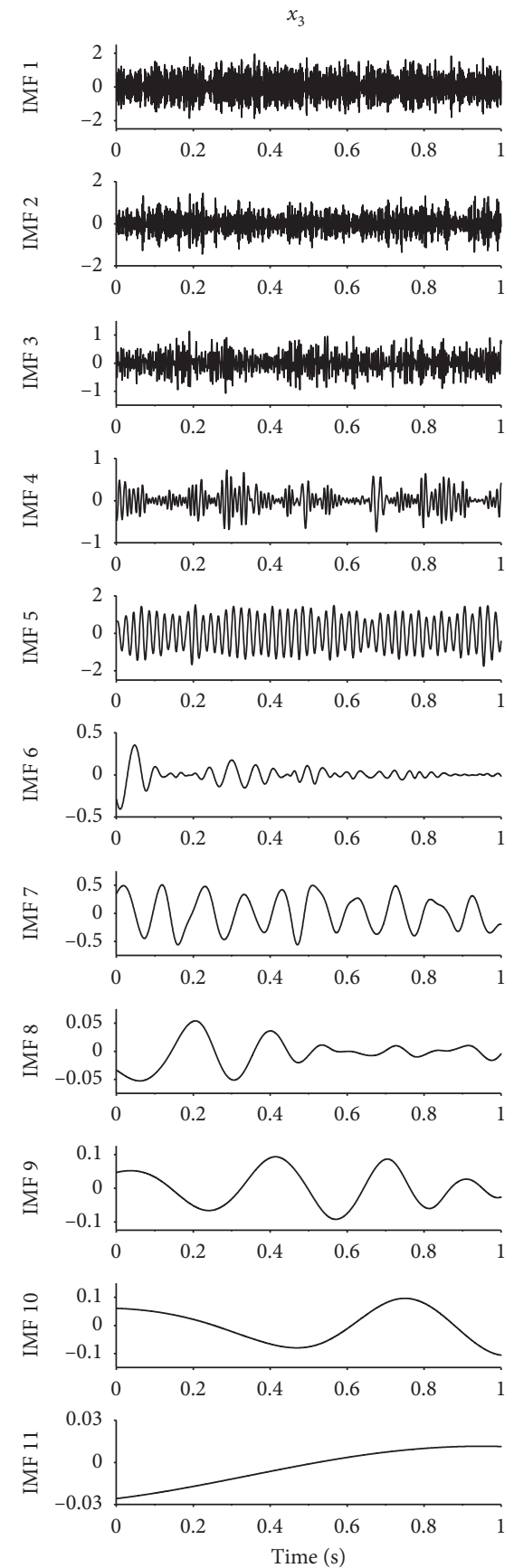

(c)

FIgURE 3: MEMD-based decomposition of simulation signal.

$$
z_{j}^{(c)}=\operatorname{int}\left(c y_{j}+0.5\right)
$$

where $c$ is the number of categories; int is the rounding function.

(3) Calculate the embedding vector $z_{i}^{(m, c)}$ using the following formula:

$z_{i}^{(m, c)}=\left(z_{i}^{(c)}, z_{i+d}^{(c)}, \cdots, z_{i+(m-1) d}^{(c)}\right), \quad i=1,2, \cdots, N-(m-1) d$, where $m$ is embedding dimension; $d$ is time delay.

(4) Calculate dispersion $\pi_{v_{0} v_{1} \cdots v_{m-1}}(v=1,2, \cdots, c), \quad$ where $\quad z_{i}^{(c)}=v_{0}$, $z_{i+d}^{(c)}=v_{1}, \cdots$, and $z_{i+(m-1) d}^{(c)}=v_{m-1}$. The number of possible dispersion patterns is equal to $c^{m}$.

(5) For each potential dispersion pattern, the relative frequency is calculated by 


$$
P\left(\pi_{v_{0} v_{1}, \cdots, v_{m-1}}\right)=\frac{\operatorname{num}\left(\pi_{v_{0} v_{1}, \cdots, v_{m-1}}\right)}{N-(m-1) d},
$$

where num $\left(\pi_{v_{0} v_{1}, \cdots, v_{m-1}}\right)$ denotes the number of $z_{i}^{(m, c)}$ mapped to $\pi_{v_{0} v_{1}, \cdots, v_{m-1}}$.

(6) Reference the definition of Shannon's entropy; dispersion entropy is defined as follows:

$$
D E(x, m, c, d)=-\sum_{\pi=1}^{c^{m}} P\left(\pi_{v_{0} v_{1}, \cdots, v_{m-1}}\right) \ln \left(P\left(\pi_{v_{0} v_{1}, \cdots, v_{m-1}}\right)\right) \text {. }
$$

From the calculation process of dispersion entropy, we can draw a conclusion that the $\mathrm{DE}$ takes the maximum value when all dispersion patterns own the same probability. The larger the DE value, the more complicated the time series. The parameters recommendation for calculating DE is given in [23]: the embedding dimension $m$ is preferably 2 or 3 ; the number of categories $c$ is an integer from 4 to 8 ; the time delay $d$ is generally taken as 1 ; the length of time series should be greater than 2000 .

In order to choose parameter more appropriately, the influence of parameter on dispersion entropy is discussed. 50 sets of white noise and $1 / f$ noise are randomly generated, and the DE values are calculated under different parameters (the length of signal, embedding dimension, and class number). The mean and standard deviation of DE for 50 sets are as shown in Figure 4. It can be concluded as follows:

(1) As the length of signal increases, DE of white noise shows an upward trend, and the pink noise is opposite. Meanwhile, the longer the length of signal is, the smaller the standard deviation is, and the stronger the stability is.

(2) As the embedding dimension increases, DE of white noise and pink noise shows a significant upward trend. Meanwhile, the higher the embedding dimension $l$ is, the higher the standard deviation is, and the worse the stability is.

(3) As the class number increases, the trend of DE value is similar to embedding dimension.

Taking the abovementioned factors and actual conditions into account, this paper selects parameters as follows: the length of signal $L=10000$, embedding dimension $m=2$, and class number $c=4$.

\section{Scheme of Fault Diagnosis}

Based on the above algorithm, a novel method for fault diagnosis of engine misfire is proposed, which is divided into four steps as shown in Figure 5. Firstly, the multivariate signals can be collected by multiple sensors and acquisition system from different position of machine. Then, the MEMD is applied to multivariate signals adaptively, which can obtain IMF groups with the same frequency components in the same order. After decomposition, reconstructed signals are composed of IMF components with large correlations of the original signals, and the feature can be extracted from it. Finally, as an outstanding classifier in small samples, support vector machine (SVM) is introduced to train the model to achieve fault recognition.

\section{Misfire Test and Fault Diagnosis}

5.1. Test and Signal Acquisition. In order to verity the superiority of the proposed algorithm, the misfire fault test was carried out on a 12-cylinder diesel engine with four-stroke. The core parameters of the diesel engine are as follows: cylinder bore $(180 \mathrm{~mm})$, compression ratio (13 14), rated power/speed ( $588 \mathrm{~kW} / 2200 \mathrm{rpm})$, and V-type. Acceleration sensors were installed on four different positions of cylinder head cover to measure the vibration signals of diesel engine. The positions are located on the cover of $1^{\text {st }}$ cylinder head of left row (position 1), the cover of $6^{\text {st }}$ cylinder head of left row (position 2), the cover of $1^{\text {st }}$ cylinder head of right row (position 3), and the cover of $6^{\text {st }}$ cylinder head of right row (position 4), as shown in Figure 6. The sensitivity of acceleration sensors is $10 \mathrm{mV} /\left(\mathrm{m} / \mathrm{s}^{2}\right)$. Different misfire faults were artificially set under $2200 \mathrm{r} / \mathrm{min}$ with no load, as shown in Table 1. The misfire fault was simulated by disconnection of fuel supply to one cylinder of engine. The sample frequency was set as $20 \mathrm{kHz}$, and the sampling time is $0.5 \mathrm{~s}$ for each sample, so there are 10000 points in each sample.

\subsection{Analysis of Data}

5.2.1. Decomposition by MEMD. The original multivariate signals under four working conditions are collected on the diesel engine bench test, and the waveforms of data in time domain are shown in Figure 7. It is hard to find rules in variation signal directly although there are some difference between different states, so we need to do further processing to variation signals.

In order to discuss the composition of signal clearly, the original multivariate signals are decomposed by EMD firstly. Taking the normal state as an example, decomposition result of multivariate signal using EMD is shown as Figure 8. The number of IMF decomposed by EMD at four positions is 13, 13,14 , and 14. There is no graph for position 1 and position 2 on IMF 14 in Figure 8. Since the internal relationship of the multivariate signals is not considered, the number of IMF components after decomposing the signal of each channel by EMD will be different. Meanwhile, 44 samples of each state are decomposed by EMD to calculate the number of IMF. As is shown in Figure 9, only four samples (two in normal state, one in neighbor cylinder misfire state, and one in far cylinder misfire state) appear the situation that the number of IMF at each point is equal, and there are different characteristic frequencies in the same order of IMF component. Therefore, the next analysis step of the IMF components of each order cannot be performed.

Then, the same multivariate signals are decomposed by MEMD, as shown in Figure 10. The number of IMF decomposed by MEMD at four positions is all 15. Similarly, all samples have the same number of IMF at each point due to the characteristics of MEMD and the same characteristic frequencies appear in the same order, which provides the basis for further analysis. 


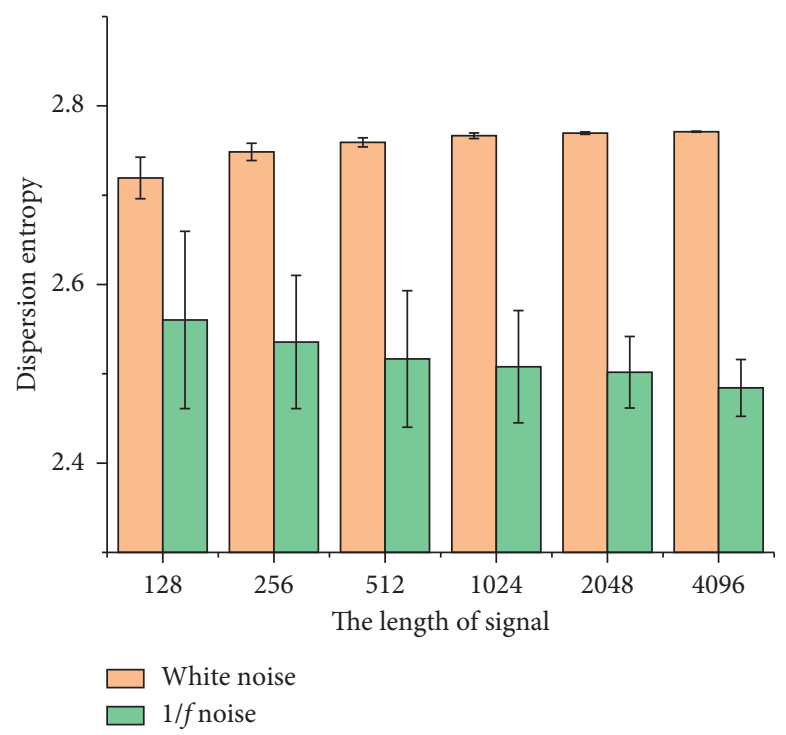

(a)

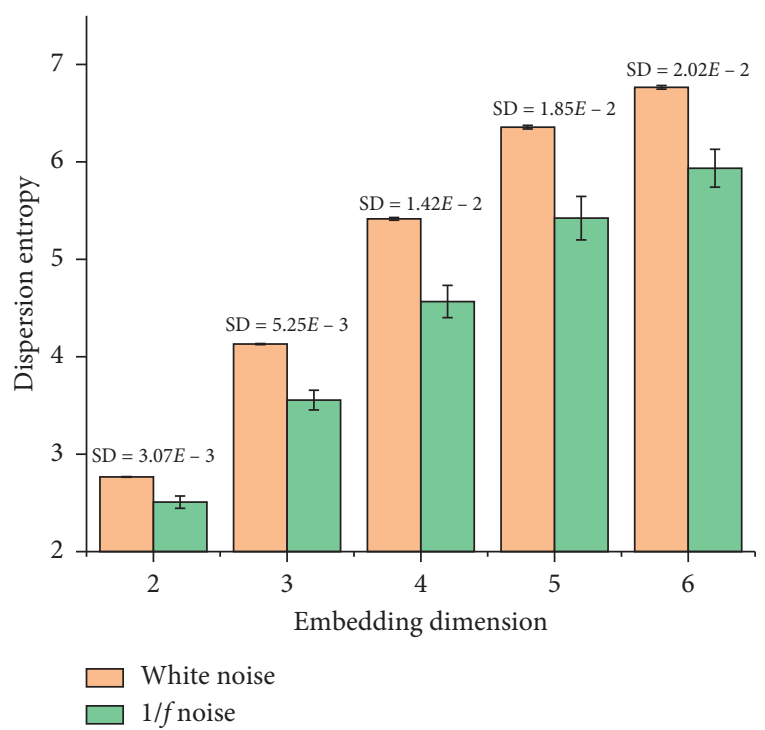

(b)

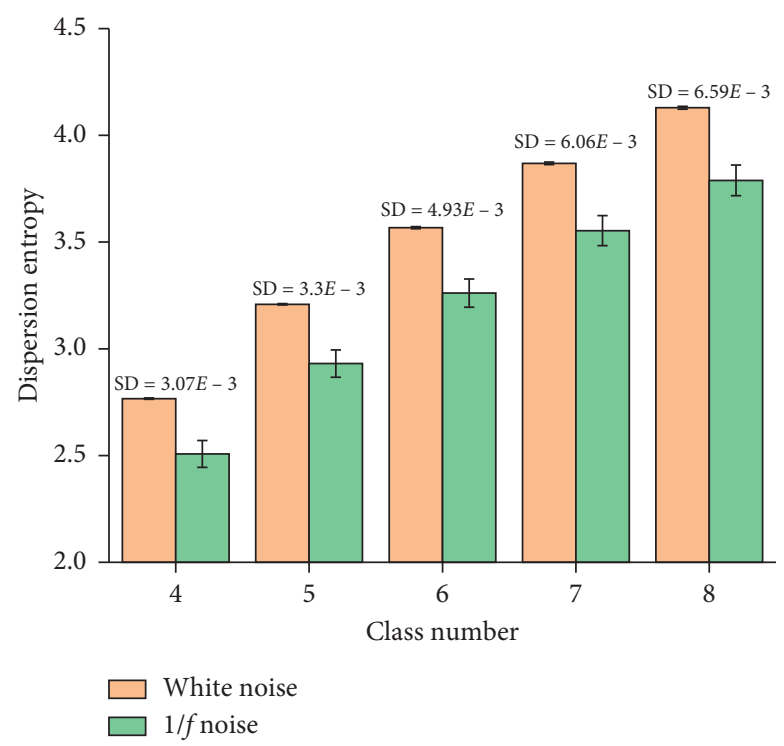

(c)

Figure 4: The mean and standard deviation of DE for 50 sets of white noise and $1 / f$ noise under different parameters. (a) Different length of signal. (b) Different embedding dimension. (c) Different class number.

In addition, taking the normal state as an example, comparing the correlation coefficient between original and IMFs in Figure 11, it can be seen that the correlation coefficients between original and IMFs decomposed by MEMD are mostly higher than the correlation coefficients by EMD and also own the smaller standard deviation and the stronger stability. Thus, the MEMD for analyzing multiple signals has significant advantages.

5.2.2. Feature Extract by Dispersion Entropy. In the data analysis, the correlation between two signals can be considered on the situation that correlation coefficient is greater than 0.3. The higher the correlation coefficient is, the stronger the correlation is. It can be seen from Figure 11 that the correlation coefficient at four positions between original signal and IMFs decreases as the IMF order increases, and correlation coefficient of first $4^{\text {th }}$ order IMF component is greater than 0.3 . Therefore, this paper selects first $4^{\text {th }}$ order IMF component to reconstruct signal.

The feature of each working state can be obtained by calculating dispersion entropy of reconstructed signal. In this paper, the parameters are set as embedding dimension $\mathrm{m}=2$, class number $\mathrm{c}=4$, time delay $\mathrm{d}=1$. The 44 sets of dispersion entropy on different position under each state are calculated, shown as Figure 12. Some conclusions can be seen that the DE values of different positions are roughly equal when the diesel engine is working properly, while $\mathrm{DE}$ values of some positions will change if misfire occurs. The $\mathrm{DE}$ value of position 1 (the cover of $1^{\text {st }}$ cylinder head of left 


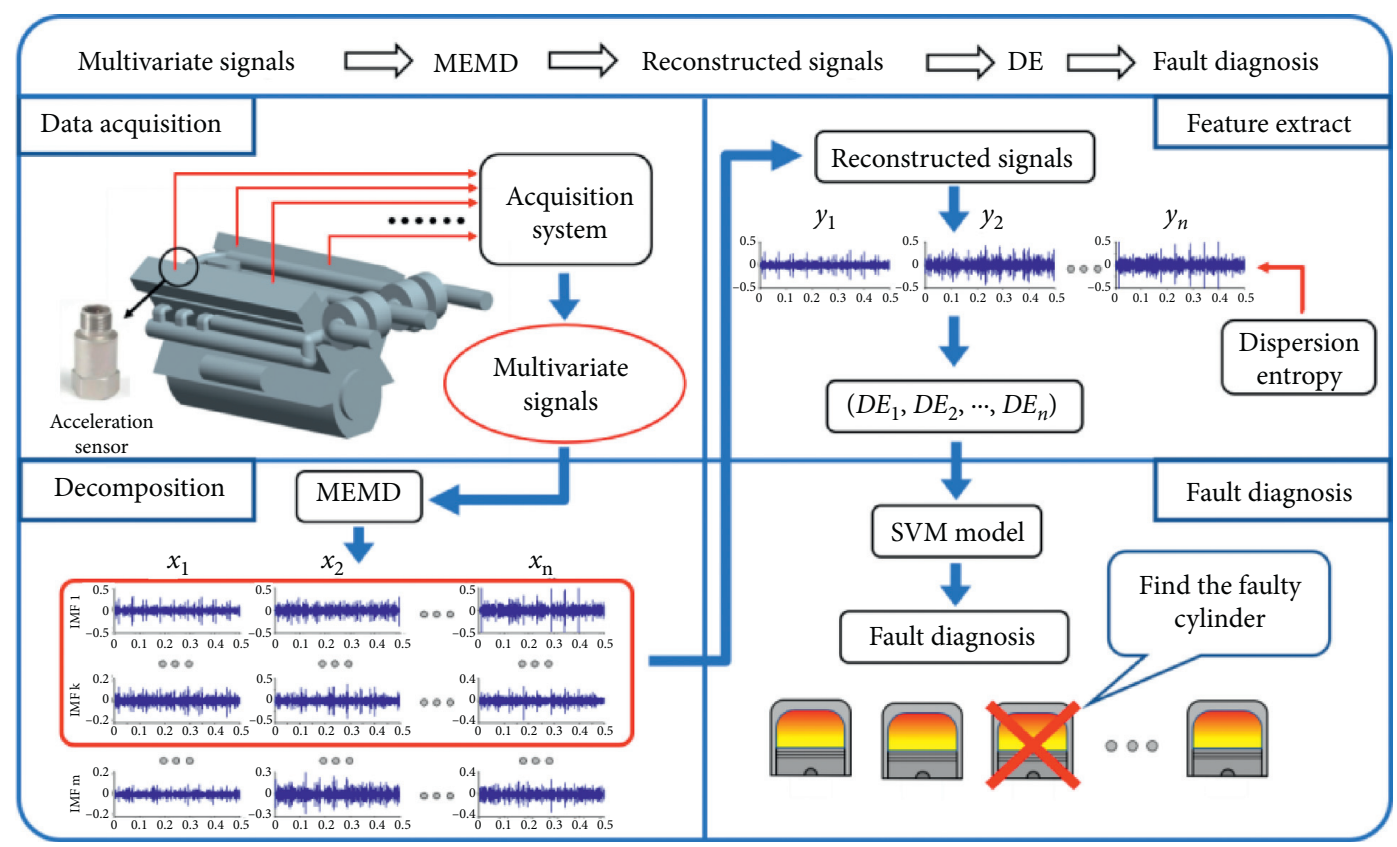

Figure 5: The fault diagnosis scheme of diesel engine.

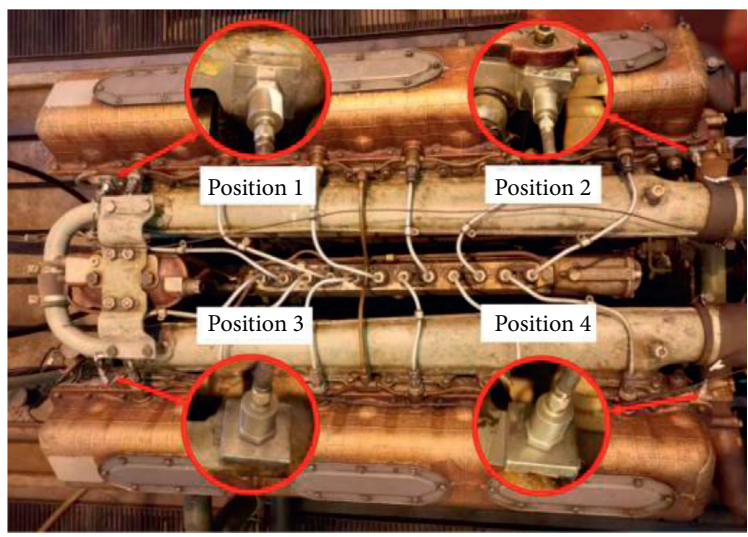

Figure 6: The measuring positions.

TABLe 1: Diesel engine misfire fault states.

\begin{tabular}{lcc}
\hline Number & Misfire cylinder & Corresponding fault \\
\hline State 1 & None & Normal \\
State 2 & 1 st cylinder of left row & Single cylinder misfire \\
State 3 & $1^{\text {st }}$ cylinder of left row and $6^{\text {st }}$ cylinder of right row & Neighbor cylinder misfire \\
State 4 & $1^{\text {st }}$ cylinder of left row and 4t $4^{\text {cylinder of left row }}$ & Far cylinder misfire \\
\hline
\end{tabular}

row) changes significantly for the reason that the misfire cylinder contains $1^{\text {st }}$ cylinder of left row. The DE values on the four positions can be characteristic parameter to recognize the state.

5.3. Diagnosis Results of Misfire Fault. Support vector machine (SVM) is a machine learning method that deals with pattern recognition, probability estimation, and other issues, which has advantages when dealing with small sample data classification. The kernel function is the main factor determining the performance of SVM; RBF kernel function can effectively deal with nonlinear problems, so this paper selects RBF kernel function for diagnosis recognition.

According to the method in the previous section, the 44 sets of samples on four positions under each working condition are calculated, among which 20 sets are randomly selected as the training sample and the remaining 24 sets are used as the test sample. Figure 13 shows the classification results of SVM. It can be seen that both of training sample 


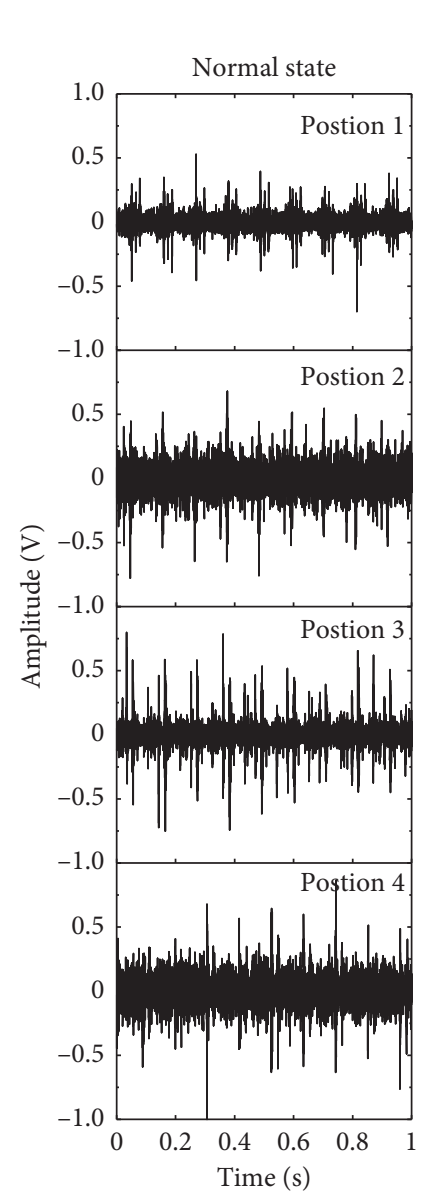

(a)

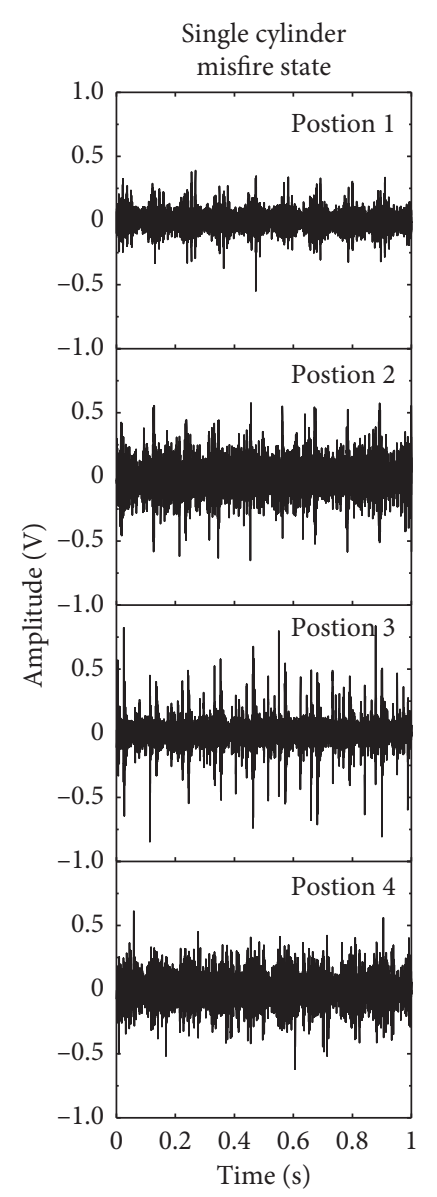

(b)

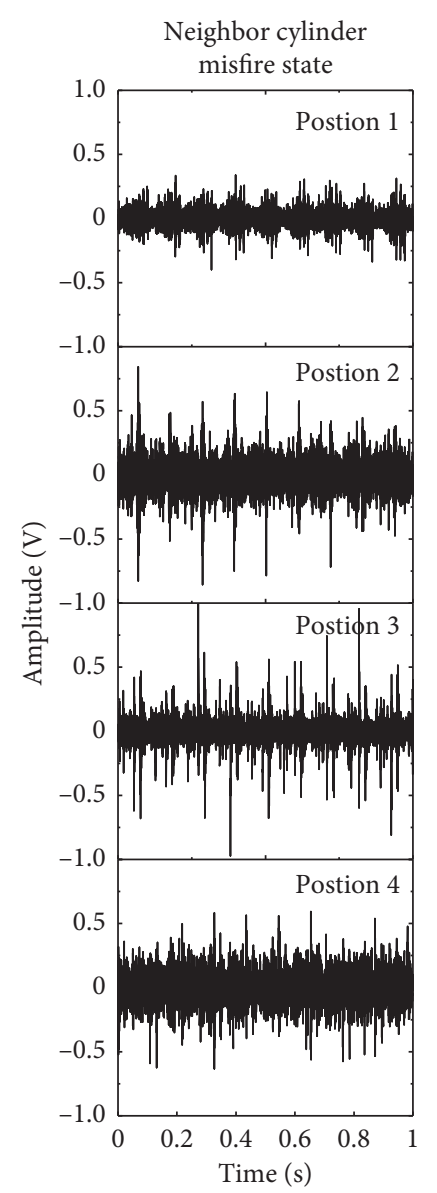

(c)

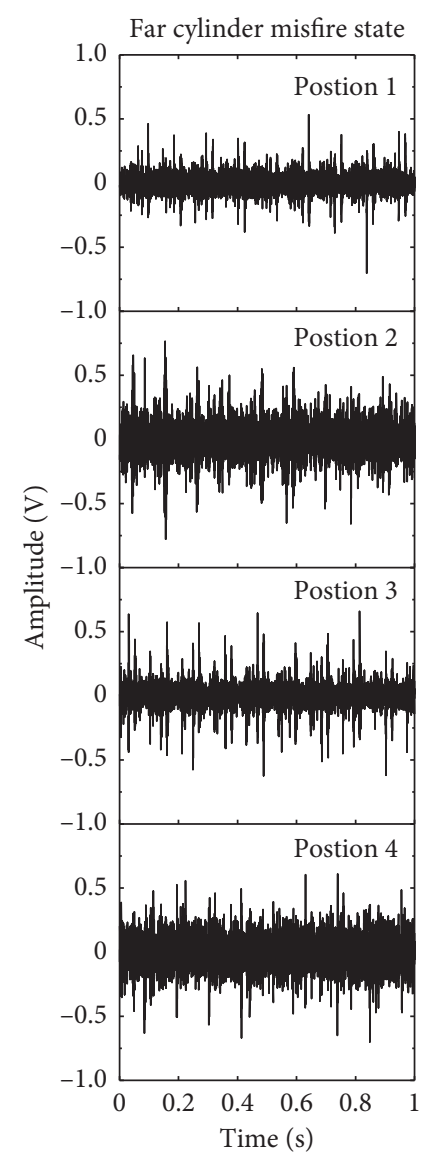

(d)

FIgURE 7: Original multivariate signals under four states.

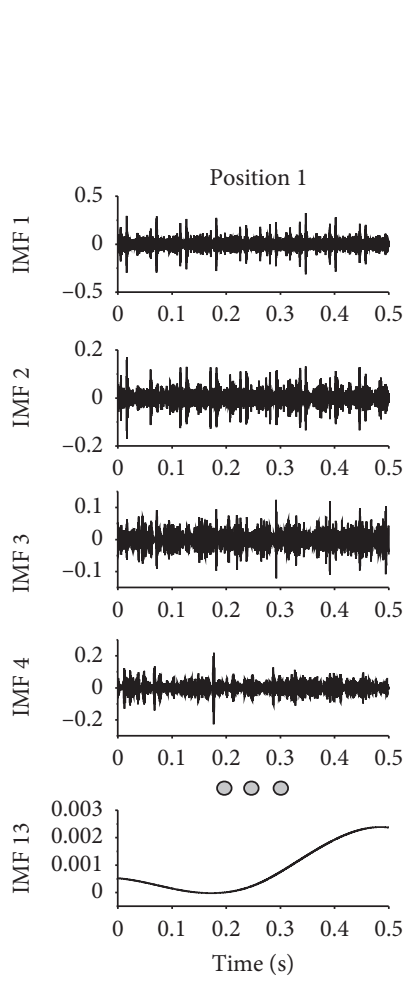

(a)
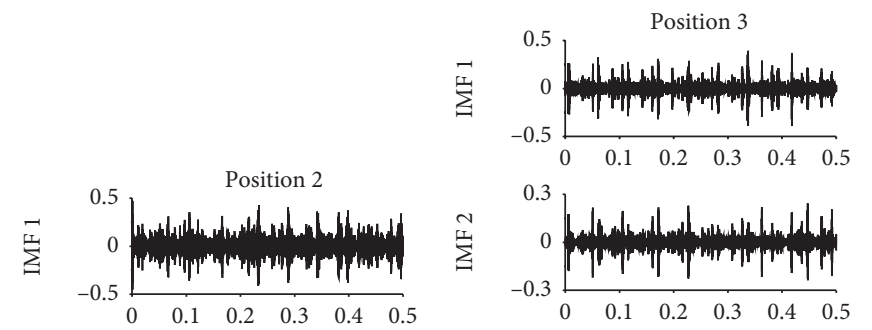

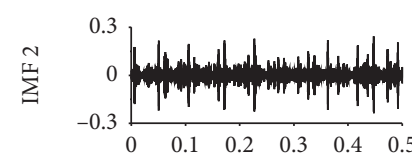
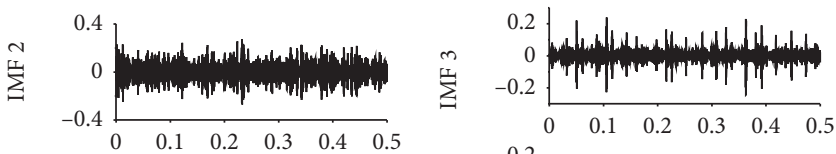

当
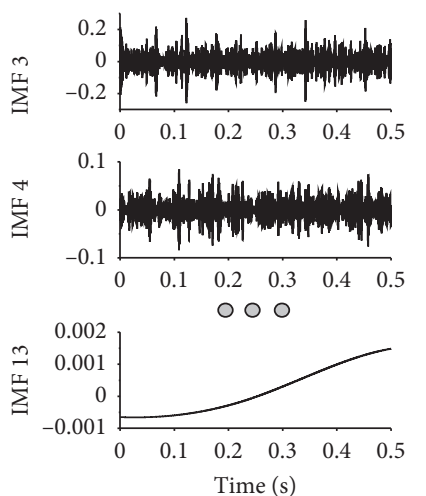

(b)
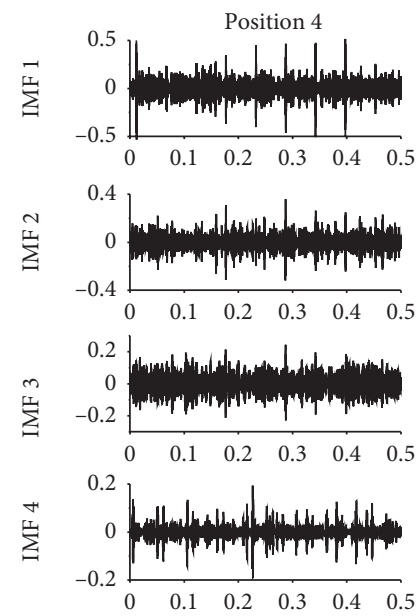

000
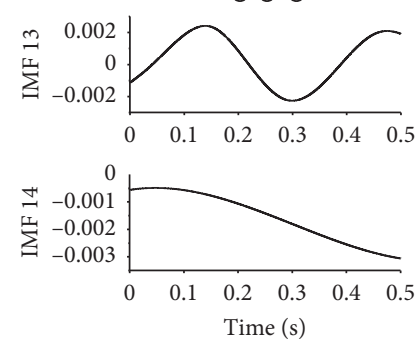

(d)

Figure 8: Decomposition result of multivariate signal using EMD (normal state). 


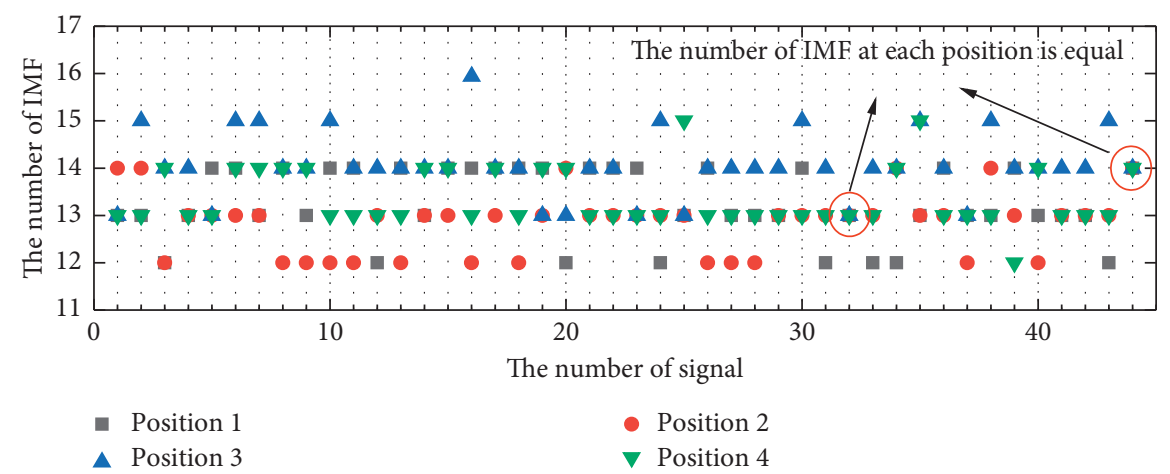

(a)

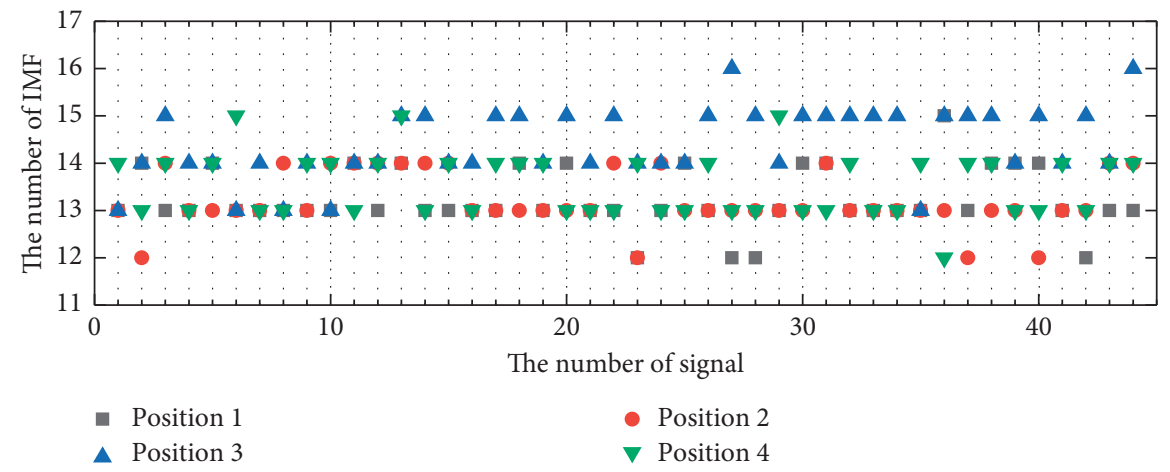

(b)

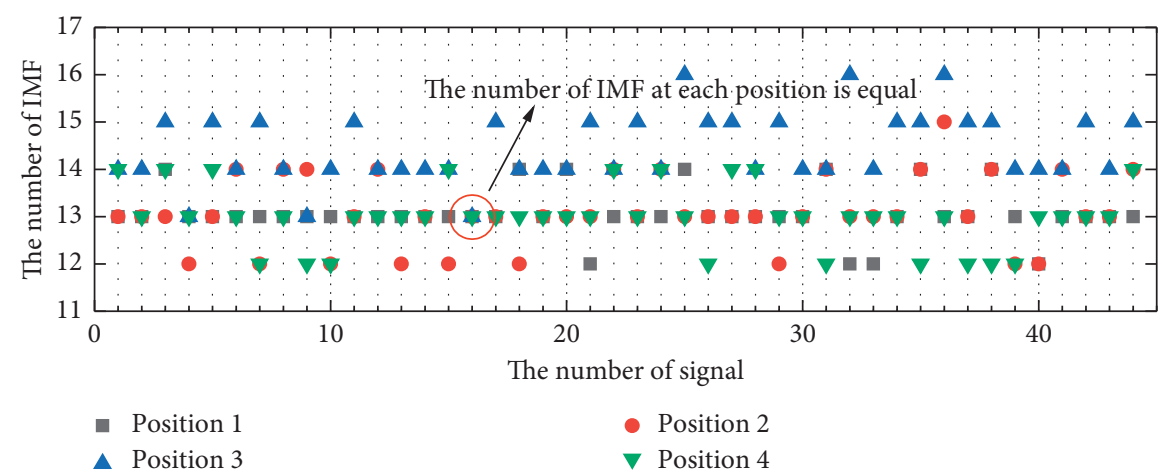

(c)

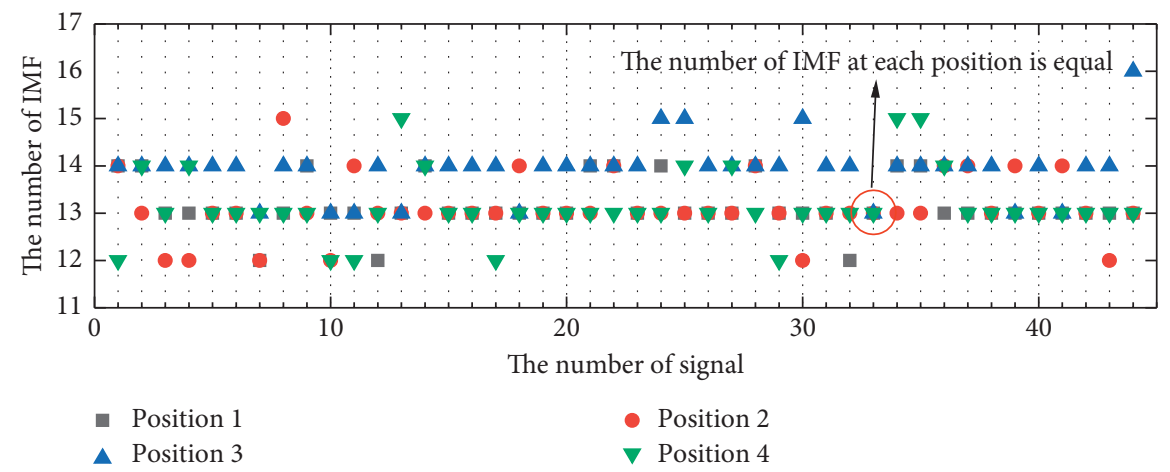

(d)

Figure 9: The distribution of IMF's number decomposed by EMD. (a) Normal state. (b) Single cylinder misfire. (c) Neighbor cylinder misfire. (d) Far cylinder misfire. 

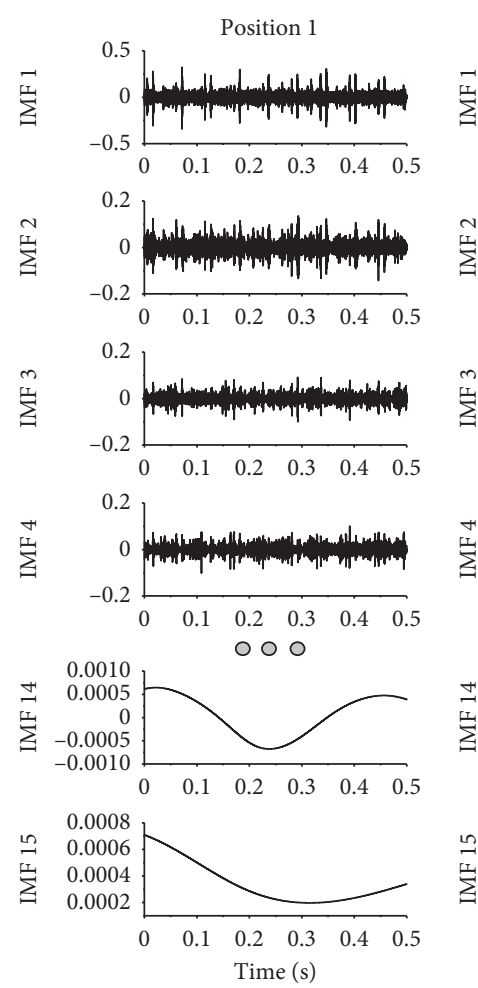

(a)
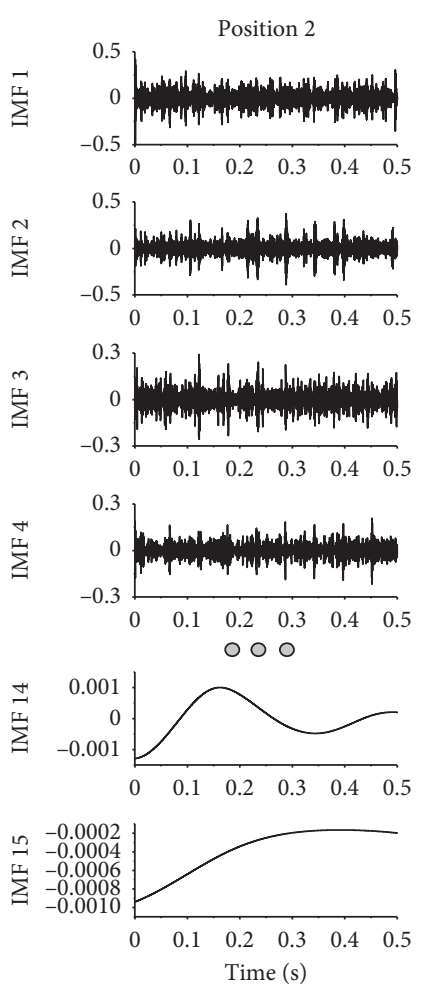

(b)
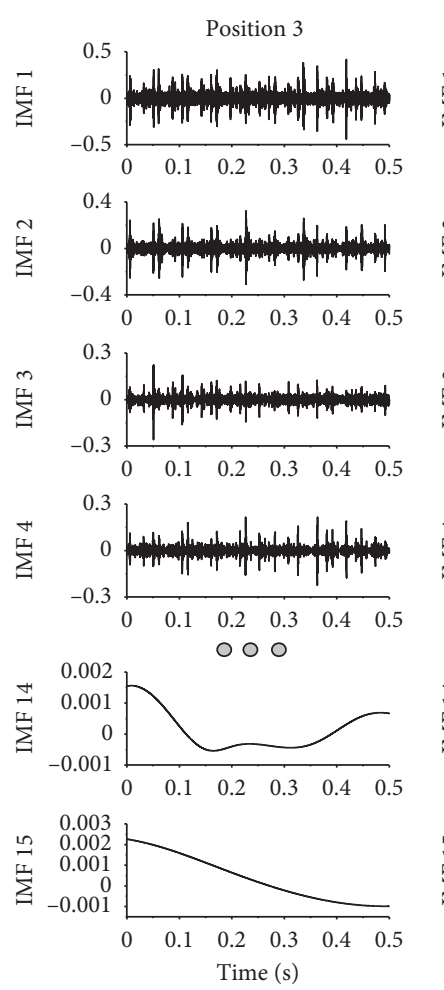

(c)
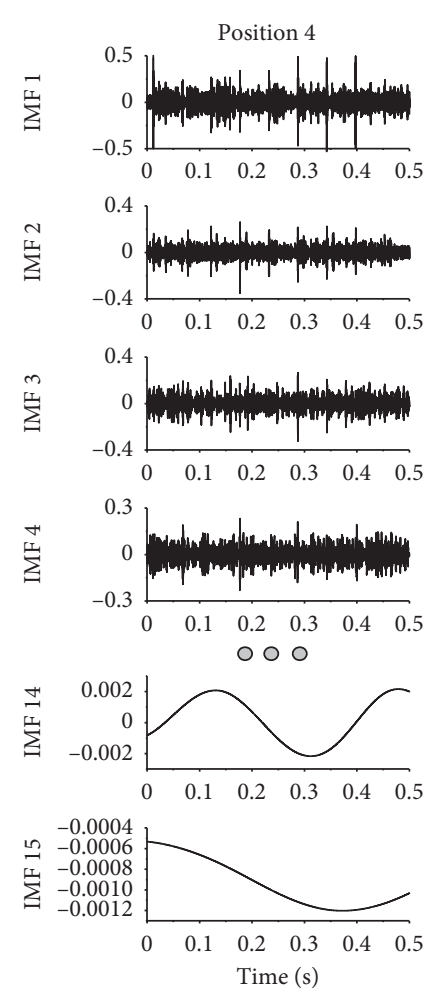

(d)

FIGURE 10: Decomposition result of multivariate signal using MEMD (normal state).

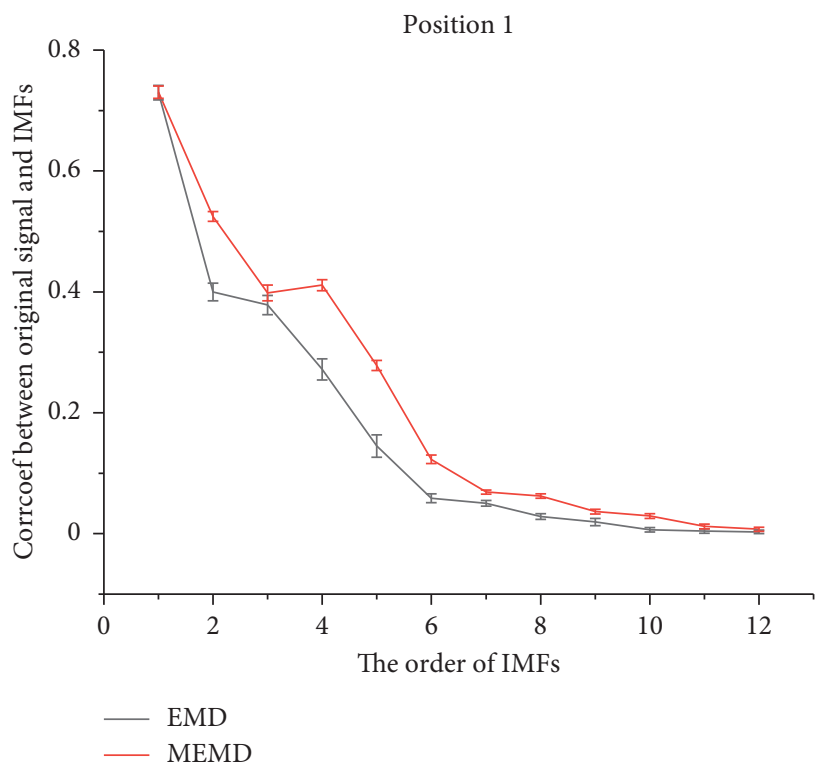

(a)

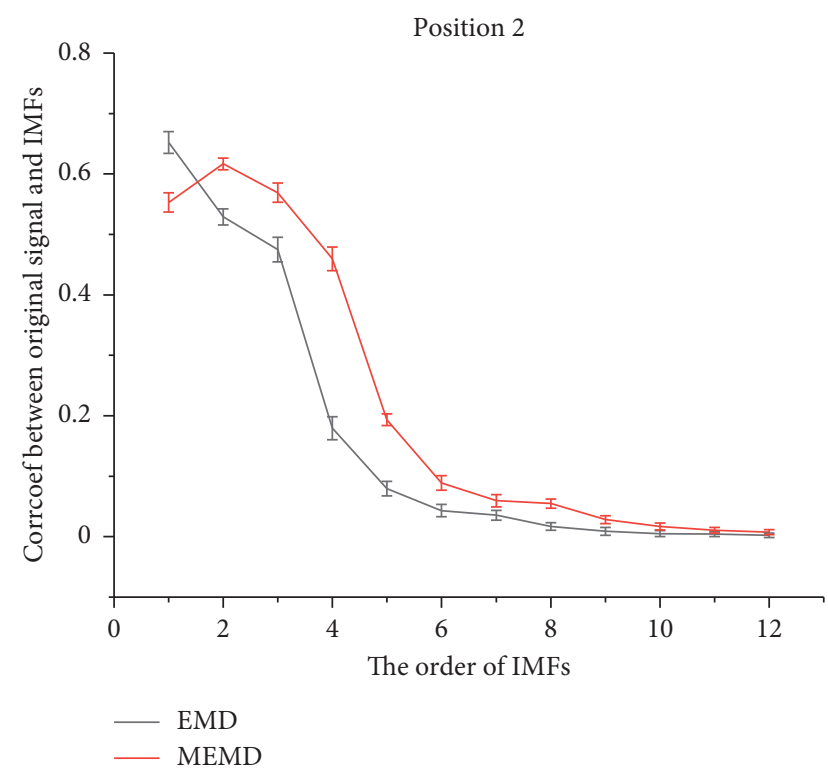

(b)

Figure 11: Continued. 


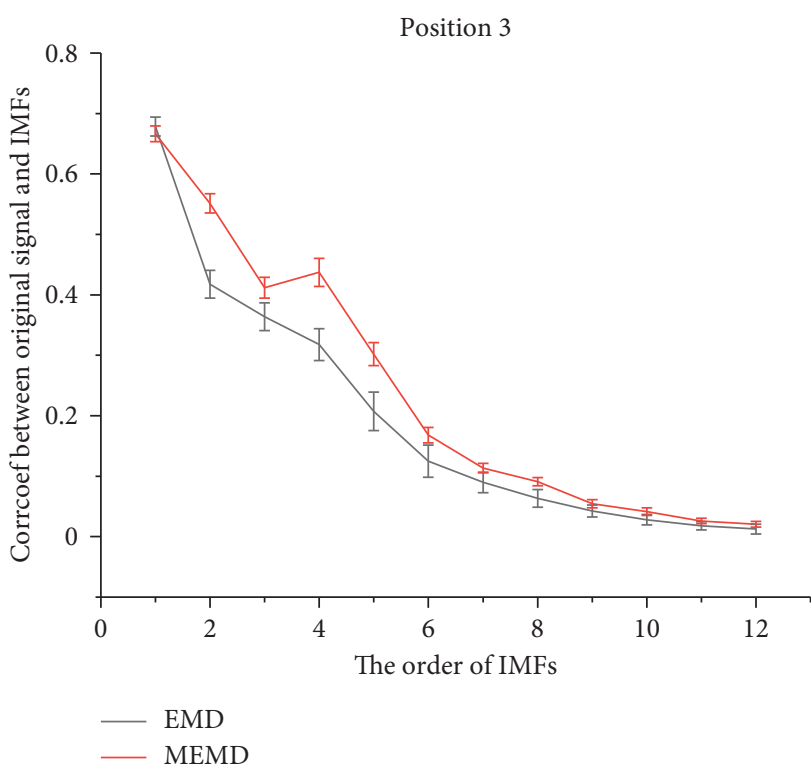

(c)

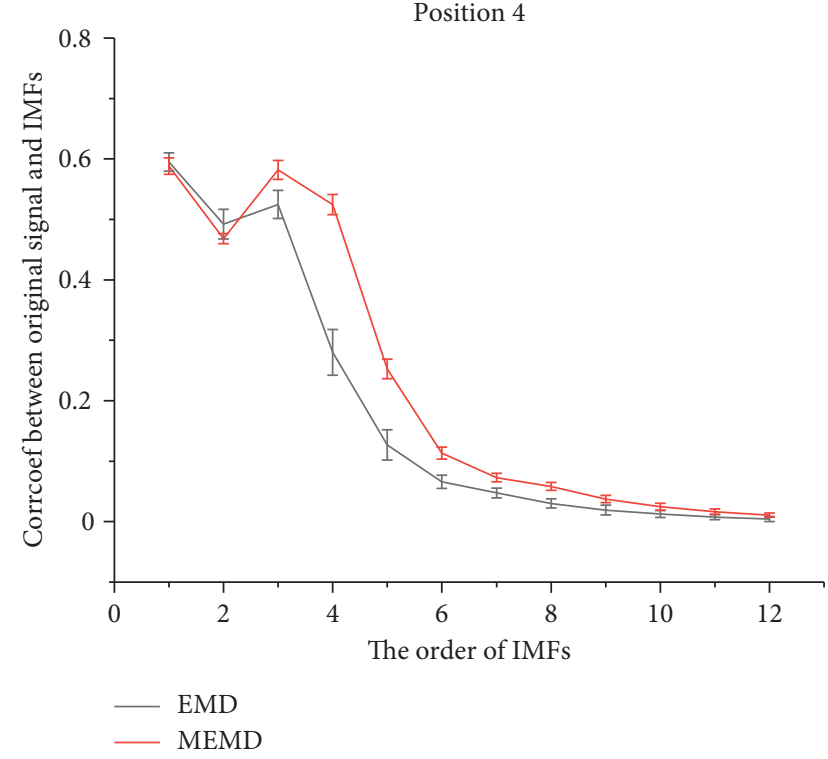

(d)

FIGURE 11: The correlation coefficient between original signal and IMFs (normal state).

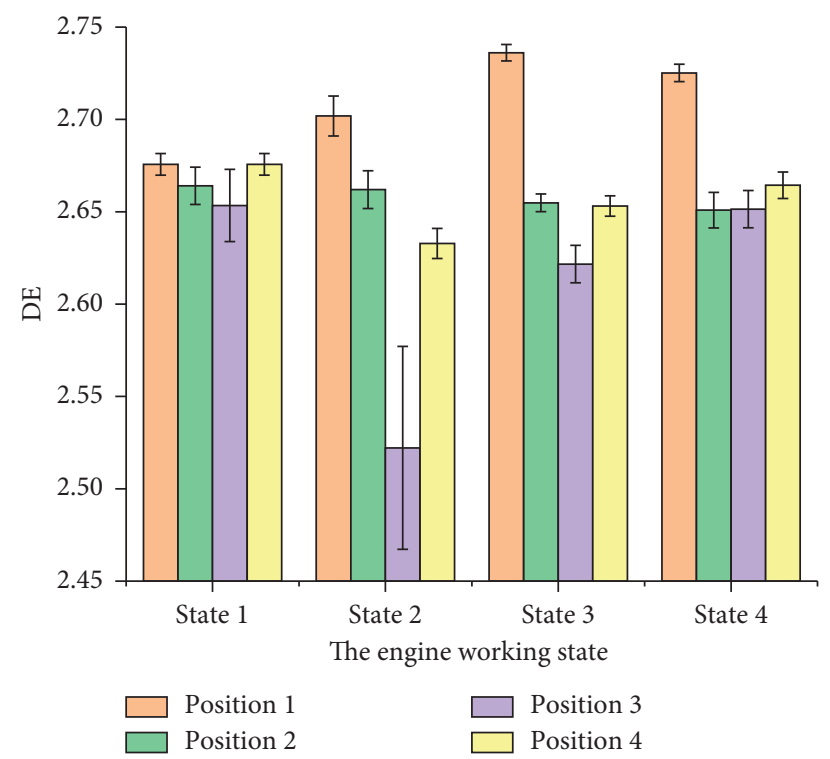

Figure 12: The mean and standard deviation of DE value on different positions under four states.

and test sample have a high recognition rate, the training sample has a far cylinder misfire set that is misclassified to the neighbor cylinder misfire set, and the test sample has a correct classification rate of $100 \%$. The results show that the method proposed by this paper can identify different types of misfire faults of diesel engine effectively and accurately.

In order to verify the superiority of the proposed method, the other three common methods are introduced to extract the fault feature of the cylinder head vibration signal, including EMD-PE, MEMD-PE, and EMD-DE. EMD-PE method represents the combination of empirical mode decomposition
(EMD) and permutation entropy (PE) and calculates the value of permutation entropy after EMD. MEMD-PE method represents the combination of multivariate empirical mode decomposition (MEMD) and permutation entropy (PE), and EMD-PE method represents the combination of empirical mode decomposition (EMD) and dispersion entropy (DE). The classification results of different methods for the same sample are as shown in Table 2. Comparing the four methods, it can be found that the total accuracy of EMD-PE, MEMD-PE, and EMD-DE is lower than the total accuracy; the MEMD-DE method performs better on identification between normal state 


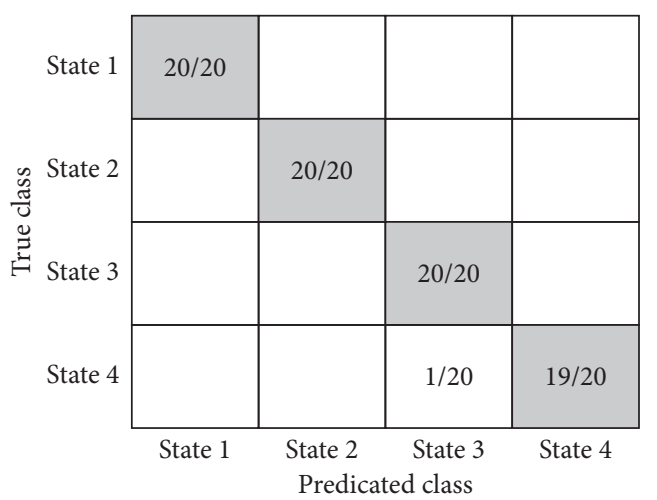

(a)

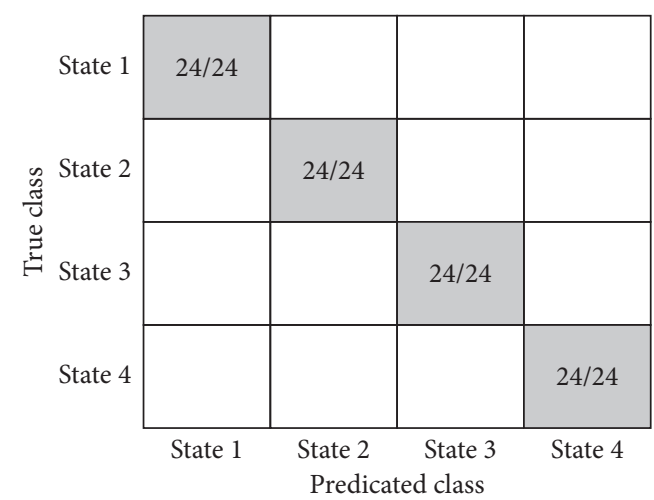

(b)

FIgure 13: Classification results of SVM. (a) Train sample. (b) Test sample.

TABle 2: Comparison of classification results of different methods.

\begin{tabular}{lccccc}
\hline \multirow{2}{*}{ Method } & \multicolumn{2}{c}{ Accuracy of each state (\%) } & \multicolumn{2}{c}{ Total accuracy (\%) } \\
& State 1 & State 2 & State 3 & 75 & 87.5 \\
EMD-PE & 83.33 & 100 & 62.5 & 96.15 & 150 \\
MEMD-PE & 79.16 & 100 & 100 & 75 & 85.42 \\
EMD-DE & 100 & 100 & 100 & 100 & 93.75 \\
MEMD-DE & 100 & 100 & & 100 \\
\hline
\end{tabular}

and different types of misfire states, while the other three methods show poor recognition ability on it.

\section{Conclusions}

In this paper, a novel diagnosis method is proposed based on MEMD and dispersion entropy, which realizes fault diagnosis accurately. The main conclusions are as follows:

(1) The multivariate signal collected from different positions of the machine is analyzed by MEMD, which obtains the same number of IMF at each point. What's more, the same characteristic frequencies appear in the same order, which illustrates that MEMD can extract the same oscillation mode from multisignals and be conducive to signal analysis further. It is an effective attempt to expand EMD to MEMD.

(2) The complexity of the cylinder head vibration signal will increase when the fire failure occurs. Compared with permutation entropy, dispersion entropy is more suitable for the extraction of nonlinear fault features due to the consideration of vibration amplitude.

(3) Compared with the other three approaches (EMD$\mathrm{PE}, \mathrm{EMD}-\mathrm{DE}$, and MEDM-PE), the fault feature extracted from MEMD-DE method is identified by SVM with a higher recognition of $100 \%$.

However, there are some problems to be solved in the future:

(1) The calculation speed of this method is flawed due to the high-dimensional operation involved. The calculation efficiency should be improved further.
(2)More types of diesel engine should be attempted. We will carry out more experiment to verify this method.

\section{Data Availability}

The data used to support the findings of this study are available from the corresponding author upon request.

\section{Conflicts of Interest}

The authors declare that they have on conflicts of interest.

\section{References}

[1] A. Sharma, V. Sugumaran, and S. Babu Devasenapati, "Misfire detection in an IC engine using vibration signal and decision tree algorithms," Measurement, vol. 50, pp. 370-380, 2014.

[2] X. Wang, C. Liu, F. Bi, X. Bi, and K. Shao, "Fault diagnosis of diesel engine based on adaptive wavelet packets and EEMDfractal dimension," Mechanical Systems and Signal Processing, vol. 41, no. 1-2, pp. 581-597, 2013.

[3] T. R. Lin, A. C. C. Tan, and J. Mathew, "Condition monitoring and diagnosis of injector faults in a diesel engine using incylinder pressure and acoustic emission techniques," Dyanmics for Sustainable Engineering, vol. 1, pp. 454-463, 2011.

[4] Z. Li, X. Yan, C. Yuan, and Z. Peng, "Intelligent fault diagnosis method for marine diesel engines using instantaneous angular speed," Journal of Mechanical Science and Technology, vol. 26, no. 8, pp. 2413-2423, 2012.

[5] S. W. Chen and Z. G. Li, "Application of grey theory in oil monitoring for diesel engine," Transactions of CSICE, vol. 23, no. 5, pp. 476-480, 2005. 
[6] X. Bi, S. Cao, and D. Zhang, "Diesel engine valve clearance fault diagnosis based on improved variational mode decomposition and bispectrum," Energies, vol. 12, no. 4, p. 661, 2019.

[7] A. Taghizadeh-Alisaraei, B. Ghobadian, T. Tavakoli-Hashin, and S. S. Mohtasebi, "Vibration analysis of a diesel engine using biodiesel and petrodiesel fuel blends," Fuel, vol. 102, pp. 414-422, 2012.

[8] J. M. Desantes, A. J. Torregrosa, and A. Broatch, "Wavelet transform applied to combustion noise analysis in high-speed DI diesel engines," SAE Technical Paper, no. 2001-01-1545, 2001.

[9] J. Hou, X. Qiao, Z. Wang, W. Liu, and Z. Huang, "Characterization of knocking combustion in HCCI DME engine using wavelet packet transform," Applied Energy, vol. 87, no. 4, pp. 1239-1246, 2010.

[10] N. E. Huang, Z. Shen, S. R. Long et al., "The empirical mode decomposition and the Hilbert spectrum for nonlinear and non-stationary time series analysis," Proceedings of the Royal Society of London. Series A: Mathematical, Physical and Engineering Sciences, vol. 454, no. 1971, pp. 903-995, 1998.

[11] Y. Li, P. W. Tse, X. Yang, and J. Yang, "EMD-based fault diagnosis for abnormal clearance between contacting components in a diesel engine," Mechanical Systems and Signal Processing, vol. 24, no. 1, pp. 193-210, 2010.

[12] M. Shahbakhti, V. Khalili, and G. Kamaee, "Removal of blink from EEG by empirical mode decomposition (EMD)," in Proceedings of the The 5th 2012 Biomedical Engineering International Conference, Ubon Ratchathani, Thailand, December 2012.

[13] A. C. Yang, S.-J. Tsai, and N. E. Huang, "Decomposing the association of completed suicide with air pollution, weather, and unemployment data at different time scales," Journal of Affective Disorders, vol. 129, no. 1-3, pp. 275-281, 2011.

[14] K. Drakakis, "Empirical mode decomposition of financial data," International Mathematical Forum, vol. 3, no. 25, 2008.

[15] G. Rilling, P. Flandrin, P. Goncalves, and J. M. Lilly, "Bivariate empirical mode decomposition," IEEE Signal Processing Letters, vol. 14, no. 12, pp. 936-939, 2007.

[16] N. Ur Rehman and D. P. Mandic, "Empirical mode decomposition for trivariate signals," IEEE Transactions on Signal Processing, vol. 58, no. 3, pp. 1059-1068, 2010.

[17] N. Rehman and D. P. Mandic, "Multivariate empirical mode decomposition," Proceedings of the Royal Society A: Mathematical, Physical and Engineering Sciences, vol. 466, no. 2117, pp. 1291-1302, 2010.

[18] P. Boguś and J. Merkisz, "Misfire detection of locomotive diesel engine by non-linear analysis," Mechanical Systems and Signal Processing, vol. 19, no. 4, pp. 881-899, 2005.

[19] H. Cui, L. Zhang, R. Kang, and X. Lan, "Research on fault diagnosis for reciprocating compressor valve using information entropy and SVM method," Journal of Loss Prevention in the Process Industries, vol. 22, no. 6, pp. 864-867, 2009.

[20] S. M. Pincus, "Approximate entropy as a measure of system complexity," Proceedings of the National Academy of Sciences, vol. 88, no. 6, pp. 2297-2301, 1991.

[21] J. S. Richman and J. R. Moorman, "Physiological time-series analysis using approximate entropy and sample entropy," American Journal of Physiology-Heart and Circulatory Physiology, vol. 278, no. 6, pp. H2039-H2049, 2000.

[22] C. Bandt and B. Pompe, "Permutation entropy: a natural complexity measure for time series," Physical Review Letters, vol. 88, no. 17, p. 174102, 2002.
[23] M. Rostaghi and H. Azami, "Dispersion entropy: a measure for time-series analysis," IEEE Signal Processing Letters, vol. 23, no. 5, pp. 610-614, 2016. 